\title{
Utility of Multidetector Computed Tomographic Angiography as an Alternative to Transesophageal Echocardiogram for Preoperative Transcatheter Mitral Valve Repair Planning
}

\author{
Craig Basman ${ }^{1}$, Caroline Ong ${ }^{1}$, Zain Kassam ${ }^{1}$, Caleb Wutawunashe ${ }^{1}$, Jennifer Conroy ${ }^{1}$, \\ Biana Trost ${ }^{1}$, Priti Mehla ${ }^{1}$, Luigi Pirelli ${ }^{1}$, Jacob Scheinerman $\mathrm{S}^{1}$, Varinder Singh $\mathrm{P}^{1}$, and \\ Chad Kliger $\mathrm{A}^{1}$ \\ ${ }^{1}$ Lenox Hill Hospital
}

February 2, 2022

\begin{abstract}
Background: Transesophageal echocardiogram (TEE) is the gold-standard for diagnosis of degenerative mitral regurgitation $(\mathrm{MR})$ and is used for guidance of transcatheter mitral valve repair (TMVr). However, TEE is an invasive diagnostic modality that requires anesthesia and esophageal intubation. Multi-detector computed tomographic angiography (MDCT) provides high resolution images and three dimensional (3D) reconstructions that allow for comprehensive assessment of complex mitral anatomy. We hypothesized that MDCT can yield similar information to 3D TEE relevant to TMVr, deferring the need for a preoperative TEE. Methods: Patients that underwent TMVr (or were evaluated for transcatheter mitral valve replacement) for degenerative MR were retrospectively analyzed from 2017 to 2019 at a single center. Patients were included in the analysis if preoperative MDCT was performed. Two experienced TEE and two MDCT readers, blinded to patient outcome and alternative imaging modality, analyzed the following characteristics: leaflet pathology (flail, degenerative, mixed), leaflet location (A1-3/P13), mitral valve area (MVA), flail width/gap, anterior-posterior (AP) and commissural diameters, posterior leaflet length, leaflet thickness, presence of mitral valve cleft and degree of mitral annular calcification (MAC). Results: Of the 87 patients, 22 had preoperative MDCT. MDCT was able to correctly identify the leaflet pathology in $77 \%(17 / 22)$. Eleven patients had a flail leaflet with 91\% (10/11) identified on MDCT and MDCT correctly predicted the dysfunctional leaflet location in 95\% (21/22). Measurements were not significantly different for MVA, flail width, commissural diameter, AP diameter, posterior leaflet length and leaflet thickness. However, measurements on MDCT were significantly overestimated for flail gap compared to TEE. Degree of MAC was similar in 91\% (10/11) between imaging modalities. Conclusion: MDCT provides similar measurements to 3D TEE for preoperative TMVr planning. Further studies are required to establish novel imaging algorithms utilizing MDCT to reduce the need for preoperative TEE.
\end{abstract}

\section{Introduction}

Transcatheter mitral valve repair (TMVr) with the Mitraclip (Abbott, Minneapolis, MN) has extended the population of patients eligible for mitral valve therapy, yet there is a sizable portion of patients that are not candidates based on anatomical exclusions. [1],[2],[3]. While multiple societal guidelines recommend the use of transthoracic echocardiography (TTE) for the initial identification of etiology and severity of degenerative mitral regurgitation (MR), preoperative transesophageal echocardiography (TEE) is required for anatomic screening. [4]. TEE is used to obtain information including etiology of MR, scallop location of flail/prolapse, location and severity of regurgitant jet(s), and leaflet thickening/calcification. However, TEE is a semi-invasive diagnostic test that requires esophageal intubation and anesthesia. Multi-detector computed tomographic angiography (MDCT) provides high-resolution images and three-dimensional (3D) reconstructions that allow for a comprehensive assessment of complex mitral anatomy [5]. We hypothesized 
that MDCT can yield similar information to 3D TEE relevant to TMVr, reducing the need for a preoperative TEE.

\section{Methods}

Patients who underwent TMVr or were evaluated for transcatheter mitral valve replacement (TMVR) for degenerative MR were retrospectively analyzed from 2017 to 2019 at a single center $(n=87)$. Patients were included in the analysis if preoperative MDCT was performed $(n=22)$. (Figure 1) Clinical information was collected from the patient chart and surgical reports. This study was approved by the institutional board review at Northwell Health. Two experienced TEE (board certified and with more than 5 years' experience) and two MDCT readers (board certified and with less than 2 years' experience), blinded to patient outcomes and alternative imaging modality, analyzed the following characteristics: leaflet pathology (flail, degenerative, mixed), leaflet location (A1-3/P1-3), mitral valve area (MVA), flail width/gap, anteriorposterior (AP) and commissural diameters, posterior leaflet length, maximal leaflet thickness (measured at A2/P2 region), presence of mitral valve cleft and degree of mitral annular calcification (MAC).

Figure 1 : Flowchart illustrating patient inclusion and exclusion algorithm

\section{CT Measurements}

Patients were included only if they had retrospective, electrocardiography (ECG)-gated, MDCT prior to any mitral intervention. Readers used both 3D reconstruction and multiplanar reconstruction (MPR) to assess leaflet pathology, location, MVA, flail width/gap, anterior-posterior (AP) and commissural diameters, posterior leaflet length, maximal leaflet thickness (measured at A2/P2 region), presence of mitral valve cleft and degree of MAC.) Measurements were averaged and in cases of discrepancy between leaflet pathology/location, a $3^{\text {rd }} \mathrm{CT}$ reader was asked to evaluate as an independent 'tie breaker.' Leaflet pathology and location were assessed with a combination of volume rendered 3D and MPR views. The dimensions of the mitral annulus were calculated with MPR CT at end-diastole. Leaflet characteristics (flail width/gap, leaflet length and leaflet thickness) were calculated based on MPR. (Figure 2) Volume-rendered 3D imaging was used to assess if a cleft mitral leaflet was present. Degree of MAC was labeled as none, mild, moderate, or severe. Severity was calculated based on a previously published cardiac CT-based score [6].

\section{TEE Measurements}

Pre-procedural TEE was performed using Epiq CVx system with an X8-2t Live 3D transducer (Philips Medical System, Andover, MA). The mitral valve was imaged and evaluated by conventional 2D with multiplane acquisition, color, and spectral Doppler imaging as well as by 3D TEE. Mitral valve morphology, MVA at end diastole, coaptation depth and length, flail width, flail gap, posterior mitral leaflet length, and AP and commissural diameters were assessed and measured using 2D and 3D images with multiplanar reconstruction using Q-Lab Software (Philips). Presence and location of mitral leaflet clefts were evaluated using 3D imaging with color Doppler. Presence and severity of MAC was assessed qualitatively. Discrepancies in assessment of valvular morphology and measurements were resolved by consensus.

Figure 2: Image A shows Flail Gap measured on MDCT, and Image B reveals similar measurements on TEE.

Figure 3: Mitral valve area (MVA) measured on multiplanar reconstruction on TEE compared to MVA measured on MDCT.

Figure 4: Posterior leaflet measurement on TEE vs MDCT

\section{Statistical Analysis}

For patient characteristics, continuous variables were reported as mean with standard deviation (SD) and compared between two groups using a 2-sample independent t tests or Mann-Whitney U test (non-uniform data). $\mathrm{P}$ values [?]0.05 were considered statistically significant. All statistical analyses were performed with the use of SAS software, version 9.2 (SAS institute, Cary, NC). 


\section{Results}

Of the 87 patients, 22 had preoperative MDCT. Fifeteen patients $(68 \%)$ received TMVr, and 7 patients $(32 \%)$ did not receive TMVr.

Table I : Describes differences measured on TEE and MDCT.

\begin{tabular}{llll}
\hline & TEE & CT & P Value \\
\hline Mitral Valve Area (cm $\left.{ }^{2}+/-\mathrm{SD}\right)$ & $4.7+/-1.19$ & $4.9+/-1.63$ & $\mathrm{P}=0.73$ \\
Flail Width (mm +/- SD) & $10.9+/-3.22$ & $8.7+/-3.18$ & $\mathrm{P}=0.14$ \\
Flail Gap (mm +/- SD) & $4.43+/-3.1$ & $8.1+/-2.01$ & $\mathrm{P}=0.01$ \\
AP Diameter (mm +/- SD) & $29.7+/-5.5$ & $31+/-5.07$ & $\mathrm{P}=0.412$ \\
Commissural Diameter (mm +/- SD) & $37.01+/-6.5$ & $38.77+/-4.91$ & $\mathrm{P}=0.32$ \\
Posterior Leaflet Length (mm +/- SD) & $13.2+/-3.2$ & $13.46+/-2.43$ & $\mathrm{P}=0.77$ \\
Leaflet Thickness (mm +/- SD) & $2.1+/-0.7$ & $1.96+/-0.551$ & $\mathrm{P}=0.36$ \\
\hline
\end{tabular}

Leaflet Pathology: Flail, degenerative (non-flail), or functional

Though the cohort of patients we evaluated were classified in our registry as DMR, 6 of the 22 patients were felt to be predominantly functional in etiology by review on TEE. MDCT classified them correctly in $4 / 6$ cases $(66 \%)$. Of the 16 cases that were analyzed as degenerative etiology, there were 7 patients with non-flail (most commonly prolapse) and 11 cases of flail pathology. MDCT correctly identified all 11 cases of flail pathology. Of the 7 non-flail cases, there were 2 cases $(28.6 \%)$ that were incorrectly identified as flail pathology on MDCT (on TEE both cases were labeled as prolapse but not flail)

\section{Leaflet Location}

MDCT correctly predicted the dysfunctional leaflet location $95 \%(21 / 22)$ of the time. There were 3 cases in which degenerative dysfunction occurred in more than one location. MDCT was able to determine the major dysfunction (flail) in each of them but did not to pick up the secondary area of degeneration. In one patient with a functional MR, it appeared that there was prolapse of one segment on MDCT. In combination, MDCT was able to correctly identify the leaflet pathology and location in $77 \%$ (17/22) of patients.

Mitral Valve Area, AP/commissural dimensions, and MAC

The mean MVA was $4.7+/-1.19 \mathrm{~cm} 2$ on TEE vs $4.9+/-1.63$ on MDCT $(\mathrm{p}=0.73)$. Both AP diameter $(29.7+/-5.5 \mathrm{~mm}$ vs $31+/-5.07 \mathrm{~mm}, \mathrm{P}=0.4)$ and commissural diameter $(37.01+/-6.5 \mathrm{~mm}$ vs $38.77+/-$ $4.91 \mathrm{~mm}, \mathrm{P}=0.3)$ were similar between TEE and MDCT. Degree of MAC was also similar in $91 \%$ with MAC underestimated on TEE in 2 patients (Table II).

Table II : Degree of Mitral Annular Calcification

\begin{tabular}{lll}
\hline Patient \# & MAC Degree (TEE) & MAC Degree (MDCT) \\
\hline 1 & none & minimal \\
2 & mild & mild \\
3 & mild & moderate \\
4 & none & none \\
5 & mild & large \\
6 & none & none \\
7 & none & minimal \\
8 & none & none \\
9 & none & none \\
10 & none & none \\
11 & none & none
\end{tabular}




\begin{tabular}{lll}
\hline Patient \# & MAC Degree (TEE) & MAC Degree (MDCT) \\
\hline 12 & none & none \\
13 & none & none \\
14 & mild & moderate \\
15 & none & none \\
16 & moderate & mild \\
17 & mild & mild \\
18 & mild & mild \\
19 & mild & severe \\
20 & severe & severe \\
21 & none & none \\
22 & moderate & moderate \\
\hline
\end{tabular}

Flail Width, Flail Gap, Posterior Leaflet Length, Leaflet Thickness, Cleft Leaflet

In cases of flail pathology, there was no statistical difference between flail width measurements $(10.9+/-$ $3.22 \mathrm{~mm}$ vs $8.7+/-3.18 \mathrm{~mm}, \mathrm{P}=0.14)$. However, MDCT over-estimated the flail gap measurement $(3.6+/-$ $3.3 \mathrm{~mm}$ vs $8.1+/-2.01 \mathrm{~mm}, \mathrm{P}=0.023)$. Similar measurements were obtained for posterior leaflet length (13.24 $+/-3.2 \mathrm{~mm}$ vs $13.46+/-2.43 \mathrm{~mm}, \mathrm{P}=0.77)$ and leaflet thickness $(2.1+/-0.7 \mathrm{~mm}$ vs $1.96+/-0.551, \mathrm{P}=0.36)$. There were two cases of cleft leaflet and MDCT was unable to detect a cleft leaflet in 1 of the patients.

\section{Discussion}

To our knowledge, this paper is the first comparison of MDCT to TEE for the purpose of TMVr planning. Current generation MDCT scanners, with at least 64-slice technology capable of generating images with submillimetric spatial resolution allow for comprehensive evaluation of complex mitral anatomy. Quantitative information including leaflet length and mitral apparatus dimensions can be obtained with MPR, while volume rendering depicts a $3 \mathrm{D}$ assessment of the valve. In our study, we found that patients evaluated for TMVR/r with MDCT had similar findings of the mitral valve and valvular apparatus compared to TEE.

Our results found that measurements between MDCT and TEE were not significantly different for MVA, flail width, commissural diameter, AP diameter, posterior leaflet length and leaflet thickness. Previously, Fuechtner et al found that MDCT can diagnose mitral valve prolapse with high accuracy. Our study also found that MDCT can accurately identify the pathological leaflet scallop location, discriminate between flail vs billowing leaflets and characterize leaflet thickening. Shanks et al, also found that 3D TEE has comparable mitral valve geometry to MDCT (including similar posterior leaflet length measurement). Sizing of the mitral annulus is traditionally done with MDCT, though our study found that measurements were similar among imaging modalities. Additionally, annular calcification is best assessed with MDCT, yet MAC severity was also comparable.

We did find a discrepancy of the flail gap measurements. It is unclear why MDCT tended to overestimate the flail gap. Prior studies have felt that the four-chamber view on MDCT resulted in an overestimation of billowing in prolapsed valves [5]. Our CT readers therefore used the two- and three- chamber reformations on MDCT to measure flail gap, however we still found a significant difference. We cannot rule out the possibility that MDCT is more accurate than TEE, and perhaps we did not appreciate the full extent of flail on TEE. Mitral regurgitation may be a dynamic process and the volume load (with IV contrast) for MDCT along with the sedation given during TEE may also lead to real life changes in the mitral pathology measurements. We therefore can't exclude the possibility that the differences found were accurate. Identification of cleft leaflet is best recognized with 3D TEE. There were two patients in our study with cleft leaflet, and MDCT was only able to detect the cleft in one of them. The slit-like appearance of a cleft leaflet can be difficult to see on 2D TTE or TEE. Cleft leaflets are identified on 3D TEE. With MDCT, using MPR projections is difficult to assess cleft leaflet, but with VR 3D imaging we were able to identify one of the cleft leaflets. 
However, adjusting the window of the 3D rendered image can make assessment of thin/soft tissue structures of the mitral leaflet a challenge, particularly clefts or pseudoclefts.

\section{Limitations}

As with most imaging studies, our study is limited by inter-observer bias. Interestingly, our MDCT readers were less experienced than the TEE readers and still were able to generate similar measurements. Despite our high-quality scanner there was sometimes systolic artifact on MDCT which may reduce accuracy. Additionally, in cases where leaflet pathology involves more than one area, interpretation on MDCT may be limited. Since obtaining preoperative MDCT is not standard practice for DMR we had a limited number of patients included and the potential for selection bias of those included. Most commonly MDCT was obtained in patients with complex TEE anatomy to further assess the mitral valve apparatus. Lastly, the imaging was performed on different days and the hemodynamics may vary among TEE (done under sedation) and MDCT (performed with IV contrast volume load).

\section{Clinical Utility}

In the era of SARS-COV2, limiting procedures, pre-procedural testing and visits is particularly important. Ideally, in patients with normal kidney function, combining transthoracic echocardiographic information with MDCT would potentially avoid the need for preoperative TEE (an invasive test which requires sedation and esophageal intubation). Furthermore, MDCT can then be used for adjunctive preoperative coronary evaluation.

\section{Conclusions}

MDCT provides similar measurements to 3D TEE for comprehensive assessment of mitral valve anatomy. Combining MDCT with TTE may produce the necessary information for TMVr planning and avoid the need preoperative TEE. Further studies are required to establish novel imaging algorithms utilizing MDCT without the need for preoperative TEE for transcatheter mitral valve therapy.

\section{References}

1. Feldman T, Foster E, Glower DD, et al. Percutaneous repair or surgery for mitral regurgitation. N Engl J Med. 2011 Apr 14;364(15):1395-406. Epub 2011 Apr 4. Erratum in: N Engl J Med. 2011 Jul 14;365(2):189. Glower, Donald G [corrected to Glower, Donald D].

2. Privitera S, Butany J, Cusimano RJ et al. Alfieri Mitral Valve Repair. Circulation. 2002;106:e173-e174

3. Maisano F, La Canna G, Colombo A, et al. The evolution from surgery to percutaneous Mitral Valve Interventions: the role of the edge-to-edge technique. Journal of the American College of Cardiology 2011: 58:2174.

4. Otto CM, Nishimura RA, Bonow RO, et al. 2020 ACC/AHA Guideline for the Management of Patients with Valvular Heart Disease: Executive Summary: A Report of the American College of Cardiology/American Heart Association Joint Committee on Clinical Practice Guidelines [published correction appears in Circulation. 2021 Feb 2;143(5):228] [published correction appears in Circulation. 2021 Mar 9;143(10):784]. Circulation . 2021;143(5):35-71

5. Guerreiro C, Fonseca C, Ribeiro J, et al. Isolated Cleft of the Posterior Mitral Valve Leaflet: The Value of 3DTEE in the Evaluation of Mitral Valve Anatomy. Echocardiography . 2016:33(8):1265-1266

6. Guerrero M, Wang DD, Pursnani A, et al. A Cardiac Computed Tomography-Based Score to Categorize Mitral Annular Calcification Severity and Predict Valve Embolization. JACC Cardiovasc Imaging . 2020:13(9):1945-1957.

7. Feuchtner GM, Alkadhi H, Karlo C, et al. Cardiac CT angiography for the diagnosis of mitral valve prolapse: comparison with echocardiography. Radiology . 2010:254(2):374-83.

8. Shanks M, Delgado V, Ng AC, et al. Mitral valve morphology assessment: three-dimensional transesophageal echocardiography versus computed tomography. The Annals of Thoracic Surgery . 2010:90(6):1922-9.

\section{Hosted file}


CTA for TMVr Planning Figures.docx available at https://authorea.com/users/458609/articles/ 555106-utility-of-multidetector-computed-tomographic-angiography-as-an-alternativeto-transesophageal-echocardiogram-for-preoperative-transcatheter-mitral-valve-repairplanning 